\title{
Discussion on Uncertainty Management in Power Systems
}

\author{
Omid Alizadeh Mousavi, Rachid Cherkaoui \\ École Polytechnique Fédérale de Lausanne (EPFL)
}

This discussion is deliberated regarding the presentations of the session on "managing uncertainty in power systems" in $9^{\text {th }}$ IREP Symposium 2013.

The power grids are continuously exposed to numerous sources of uncertainties which threaten the reliable and secure supply of electricity. These uncertainties are mainly due to the occurrence of contingencies and more recently the high penetration of intermittent renewables. Managing uncertainties in power systems regarding the system security has received many attentions from power engineering communities using deterministic (e.g. $\mathrm{N}-1$ criteria) and probabilistic (e.g. stochastic and robust) optimization approaches. These optimization methods aim to obtain a balance between the economy and security. In this respect, the value of security can be assessed in terms of risk. The risk is defined as the sum of the product of the consequence and probability of a given set of events. The evaluation of risk should be performed carefully in order to consider the high-probability/low-impact scenarios as well as the low-probability/high-impact ones. It is worth noting that the evaluation of the risk of low probable scenarios, with a certain accuracy level, is computationally much more difficult and time consuming.

In order to elucidate the issue of risk evaluation more in depth, we would like to refer to the definitions of robustness and resilience in power systems [1]. The robustness is defined as the ability of a system to maintain its function when it is subjected to a given class of perturbations, whereas the resilience is defined as the ability of a system to gracefully degrade its function in an agile way when it is subjected to a class of unexpected extreme perturbations. Therefore, the solution of an optimization problem should provide a tradeoff between the robustness and resilience.

So far in the planning and operation optimizations, the resilience related events are not considered since the obtained solution becomes more expensive from the economical point of view and also its computational complexity increases. But it should be noted that when a power system is designed to be robust to a specific class of perturbations, it becomes more vulnerable to another class of failures. Similarly, a power system that is resilient to a certain type of failure may be fragile to another one [1].

Furthermore, the analysis of the times series of blackouts size measures worldwide has shown a power law region in distributions of different quantities. It implies that the blackouts of different scales may take place and the extreme events cannot be overlooked. The system may experience large blackouts with certain probabilities and the occurrences of small/large blackouts are not independent but correlated each other [2].

In the proposed optimizations of the literatures for the management of uncertainties in power systems, the effectiveness of the obtained solutions is usually evaluated using Monte Carlo Simulation for which the dependent and cascading outages are disregarded. Since the cascading events which can result into large blackouts are neglected, the risk of the rare but large blackouts is not evaluated properly. Thus, these significant perturbations are not taken into consideration in the optimizations neither their effects are evaluated appropriately. As a result, the value of risk is not assessed effectively in the proposed optimizations for the management of uncertainties.

The consideration of the extreme perturbations in the optimizations may not be a plausible approach to solve the above-mentioned problem. However, the response of the solutions of proposed optimizations to both robust and resilient perturbations can be assessed using a variant of Monte Carlo Simulation which considers the model of dependent and cascading outages.

\section{References}

[1] L. Mili, "Taxonomy of the Characteristics of Power System Operating States", Proceeding of $2^{\text {nd }}$ NSF-RESIN workshop, January, 2011.

[2] D. E. Newman, B.A. Carreras, V.E. Lynch, I. Dobson, "Exploring complex systems aspects of blackout risk and mitigation", IEEE Transactions on Reliability, vol. 60, no. 1, pp. 134-143, 2011. 